\title{
Thyroid cancer patients' involvement in adjuvant radioactive iodine treatment decision-making and decision regret: an exploratory study
}

\author{
A. M. Sawka • S. Straus • A. Gafni • S. Meiyappan • \\ D. David • G. Rodin • J. D. Brierley • R. W. Tsang • \\ L. Thabane $\cdot$ L. Rotstein $\cdot$ S. Ezzat $\cdot$ D. P. Goldstein
}

Received: 28 April 2011 / Accepted: 25 October 2011 /Published online: 10 November 2011

(C) The Author(s) 2011. This article is published with open access at Springerlink.com

\begin{abstract}
Purpose We explored regret in thyroid cancer patients, relating to the decision to accept or reject adjuvant radioactive iodine treatment.

Methods We studied patients with a recent diagnosis of early stage papillary thyroid carcinoma, in whom treatment decisions on adjuvant radioactive iodine had been finalized. Participants completed a Decision Regret Scale questionnaire. We asked the participants to identify who made the final decision about radioactive iodine treatment. We explored the relationship between decision regret and a) degree of patient involvement in decision-making and $b$ ) receipt of radioactive iodine treatment.

Results We included 44 individuals, more than half of whom received adjuvant radioactive iodine treatment (26/ 44). Decision regret was generally low (mean 22.1,
\end{abstract}

A. M. Sawka $\cdot$ S. Meiyappan $\cdot$ D. David $\cdot$ S. Ezzat Division of Endocrinology, Department of Medicine, University Health Network-Toronto General Hospital, 200 Elizabeth Street, 12 EN-212,

Toronto, Ontario, Canada M5G 2C4

A. M. Sawka $(\bowtie) \cdot$ S. Ezzat

Division of Endocrinology, Department of Medicine,

University of Toronto,

Toronto, Ontario, Canada

e-mail: sawkaam@yahoo.com

S. Straus

Division of Geriatrics and Internal Medicine,

Department of Medicine, University of Toronto,

Toronto, Ontario, Canada

A. Gafni $\cdot$ L. Thabane

Department of Clinical Epidemiology and Biostatistics,

McMaster University,

Hamilton, Ontario, Canada standard deviation [SD] 13.0). Participants reported that the final treatment decision was made by the following: patient and doctor $(52.3 \%, 23 / 44)$, completely the patient $(27.3 \%, 12 / 44)$, or completely the physician $(20.5 \%, 9 / 44)$. Decision regret significantly differed according to who made the final decision: the patient (mean 19.0, SD 11.3), patient and doctor (mean 19.5, SD 7.4), and the doctor (mean 32.9, SD 20.37) $(F=$ 4.569; degrees of freedom $=2,41 ; p=0.016$ ). There was no significant difference in decision regret between patients who received radioactive iodine and those who did not (mean difference $-2.5 ; 95 \%$ confidence interval -10.6 , 5.6; $p=0.540$ ).

Conclusion Thyroid cancer patients who reported being involved in the final treatment decision on adjuvant radioactive iodine had less regret than those who did not.

\section{G. Rodin}

Department of Psychosocial Oncology and Palliative Care,

University Health Network and the University of Toronto,

Toronto, Ontario, Canada

J. D. Brierley $\cdot$ R. W. Tsang

Department of Radiation Oncology,

University Health Network and the University of Toronto,

Toronto, Ontario, Canada

\section{Rotstein}

Department of Surgery,

University Health Network and the University of Toronto,

Toronto, Ontario, Canada

D. P. Goldstein

Department of Otolaryngology,

University Health Network and the University of Toronto,

Toronto, Ontario, Canada 
Keywords Decision regret $\cdot$ Thyroid carcinoma Patient education $\cdot$ Medical decision making

\section{Introduction}

Thyroid carcinoma is increasingly being diagnosed [1-5], especially small primary tumors of papillary thyroid carcinoma (PTC) discovered at an early stage [3]. PTC is usually treated with total thyroidectomy, followed in some cases by adjuvant radioactive iodine (RAI) treatment [6-8]. Decision-making about adjuvant RAI treatment in early stage PTC is complex and subject to considerable medical evidence uncertainty. This uncertainty is due to contradictory findings in long-term observational studies and a lack of high quality evidence from randomized controlled trials [9]. The impact of the uncertainty of the medical evidence on patients' regret of treatment choice is not known.

According to a model of decision-justification theory, Connolly and Zeelenberg have proposed that regret is associated with the comparative evaluation of the outcome of a decision and the feeling of self-blame for having made a poor choice [10]. From this perspective, regret is regarded as an aversive emotion, in which people realize or imagine that their present situation would have been better had they decided differently in the past [10]. This regret can be experienced about the decision-making process ("process regret") and/or about the outcome of the decision ("outcomes regret") [10]. Our primary objective in this secondary study was to measure outcome regret in thyroid cancer survivors, relating to the decision to take adjuvant radioactive iodine treatment or not. Furthermore, we explored process regret by examining the relationship between patients' perceived role in radioactive iodine treatment decision-making and current regret. We also examined outcome regret, by exploring the relationship between radioactive iodine treatment status (i.e. whether this treatment was received or not) and current decision regret.

\section{Methods}

Participant recruitment

Participants in this secondary subgroup study were recruited as part of a larger study of 50 individuals diagnosed with early stage PTC, examining the effect of a computerized thyroid cancer decision aid on medical knowledge [11, 12]. Participants were required to have had primary surgical treatment for thyroid cancer (total thyroidectomy) on September 1, 2007 or later, and they participated in the study between late October of 2009 and early February of 2010. The pathology reports for all patients' thyroidectomy specimens were reviewed. Participants were required to have a primary PTC tumor $4 \mathrm{~cm}$ or smaller in diameter with no known positive lymph nodes and no known distant metastases, with no venous or lymphatic invasion, and no tall cell features, noted on surgical pathology examination $[11,12]$. We included only English-speaking individuals aged 18 years or older who had a prior thyroidectomy (completed in one or two stages), who were able to use a computer, in this study [11, 12]. Individuals with a single intra-thyroidal PTC measuring less than $1 \mathrm{~cm}$ in diameter or those who had undergone only a hemi-thyroidectomy (with no completion thyroidectomy) were not eligible $[11,12]$ since they are typically not offered RAI treatment. The measurement of decision regret was restricted to 44 participants in whom decision-making about adjuvant radioactive iodine was completed and who had either received radioactive iodine treatment or not received it (with no plans to receive it). These 44 participants had no exposure to our decision aid at the time of RAI treatment decision-making or prior to completing the decision regret questionnaire. All of these individuals received usual counseling and care from their treating physicians, relating to decision-making on radioactive iodine treatment, prior to participation in the study. The study was approved by the University Health Network Research Ethics Board, and all participants provided informed consent.

\section{Study procedure and questionnaires}

At baseline (i.e. prior to exposure to any decision aid intervention), participants were asked to complete written questionnaires on their medical history and radioactive iodine treatment decision-making. We asked the participants to identify who made the final decision for the patient to take or not take adjuvant RAI treatment, and they were offered the following options (choose one): completely my choice (with or without input from my family or friends), completely my doctor's choice, my doctor and I made the decision together (with or without input from my family or friends), someone else's choice (other than me and my doctor), or the final decision on RAI treatment has not yet been made (still thinking about it). Participants whose RAI treatment decisionmaking was completed were also asked to complete a validated decision regret questionnaire [13], specifically considering the decision to accept or reject adjuvant RAI treatment. The decision regret questionnaire is scored on a scale of 0 to 100 , with 100 representing maximal regret [13].

\section{Analyses}

The sample size of 44 respondents was primarily based on feasibility considerations, since the study was designed for exploratory or hypothesis-generation purposes. Descriptive data were presented as mean and standard deviation (SD) for 
continuous data, or number and percentage for categorical data. Respective exploratory analyses using analysis of variance (ANOVA) and an independent samples Student's $t$ test were performed, to compare decision regret according to perceived RAI treatment final decision maker (collapsed to three levels, i.e. the patient, shared decision between patient and doctor, and the doctor) and RAI treatment status (i.e. received vs. not received). Although we could not ascertain the exact date when decision-making about RAI treatment was finalized, such decisions generally follow completion of thyroidectomy. Therefore, we used the year of completion of thyroidectomy (years 2007, 2008, and 2009) as a reasonable surrogate for the time when RAI decision-making was performed. An exploratory post-hoc Pearson correlation statistic was calculated, examining the relationship between the year when thyroidectomy was completed and current decision regret, to determine whether regret tended to vary over time. The criterion for statistical significance was set at alpha $=0.05$. We did not adjust the overall level of significance for multiple testing as the analyses were primarily exploratory. Quantitative statistical analyses were performed using PASW Statistics 18.0 (IBM, Chicago, IL).

\section{Results}

Study sample

The study sample was comprised of 37 women and seven men who had been diagnosed with PTC at thyroidectomy.
The demographic characteristics of the study population are shown in Table 1. The study population represented a wide age range, and the majority had an education level higher than high school (90.9\%). More than half of the study population (26/44) had received adjuvant radioactive iodine treatment, and the rest had not received it (with no plans to receive it). More than half of the participants perceived that the final decision to accept or reject RAI treatment had been shared between themselves and their physician (23/44) (Table 1). Fewer patients perceived the decision to have been completely theirs $(27.3 \%, 12 / 44)$ or completely their treating physician's $(20.5 \%, 9 / 44)$, respectively.

Regret related to radioactive iodine treatment decision-making

In general, participants had a relatively low level of decision regret related to RAI treatment choice (mean value of 22.1, SD 13.0). However, in an exploratory analysis, decision regret was found to significantly differ according to who made the final treatment decision: the patient (mean 19.0, SD 11.3, $n=12$ individuals), shared between the patient and doctor (mean 19.5, SD 7.4, $n=23$ ), or the doctor (mean 32.9, SD 20.4, $n=9)(F=4.569$; degrees of freedom= $2,41 ; p=0.016)$. There was no significant difference in decision regret between the 26 patients who received RAI treatment (mean 21.1, SD 12.7) and the 18 who did not receive this treatment (mean 23.6, SD 13.6) (mean difference $-2.5 ; 95 \%$ confidence interval $-10.6,5.6 ; p=$ 0.540 ). We found no significant correlation between the
Table 1 Demographic characteristics of participants

\begin{tabular}{lll}
\hline Characteristic & & Number (\%) \\
\hline Age (years) & 18 to 30 & $5 / 44(11.4)$ \\
& 31 to 40 & $9 / 44(20.5)$ \\
& 41 to 50 & $17 / 44(38.6)$ \\
& 51 to 60 & $9 / 44(20.5)$ \\
Female gender & 61 or older & $4 / 44(9.1)$ \\
Received adjuvant radioactive iodine treatment & & $37 / 44(84.1)$ \\
Year thyroidectomy completed & & $26 / 44(59.1)$ \\
& 2007 & $19 / 44(43.2)$ \\
Highest level of education & 2008 & $17 / 44(38.6)$ \\
& 2009 & $8 / 44(18.2)$ \\
& High school & $4 / 44(9.1)$ \\
Who was perceived as making the final decision & College or university & $26 / 44(59.1)$ \\
on adjuvant radioactive iodine treatment & Completely patient's choice & $12 / 44(27.3)$ \\
& Completely doctor's choice & $9 / 44(20.5)$ \\
& Doctor and patient made & $23 / 44(52.3)$ \\
& decision together & \\
& Someone else & $0 / 44(0)$ \\
\hline
\end{tabular}

${ }^{\text {a }}$ Year thyroidectomy completed refers to the year when total thyroidectomy was performed (if performed in one stage) or, in the case of a two-stage thyroidectomy, to the year of the second procedure 
year of completion of thyroidectomy and current decision regret $(r=0.112, p=0.469)$.

\section{Discussion}

In this study, early stage PTC survivors had a relatively low level of decision regret relating to taking adjuvant RAI treatment or not. However, individuals who perceived that their physician made the final treatment decision on RAI treatment without their involvement reported significantly more decision regret than those who felt involved in final treatment decision-making (i.e. making the decision themselves or sharing decision-making with their physician).

Our findings are similar to those of Brehaut et al., who reported, in a study of decision-making in women regarding hormone replacement therapy, that the level of regret was significantly greater in women who indicated that their physician was the main decision-maker, compared to those who felt they were the main decision-maker or that the decision-making was shared between themselves and their physicians [13]. We did not find any relationship between the receipt of RAI treatment or not and the level of decision regret. It has been postulated that feeling regret is related to perception of lost opportunities [14, 15], so it is possible that these individuals minimized the perception of such losses. In that regard, Lin reported that prostate cancer survivors' decision regret relating to radical prostatectomy was associated with experiencing treatment side effects [16]. Furthermore, prostate cancer survivors who felt they understood the treatment and complications had significantly less regret at follow-up [16]. We did not collect data on thyroid cancer treatment side effects or patients' perceptions of feeling well-informed about treatment choice at the time of decision-making. However, we did not find any relationship between time since completion of thyroidectomy (as a surrogate of timing of RAI treatment decisionmaking) and subsequent regret, although timing of decision regret measurement was limited to within a few years after completion of thyroidectomy. Lin also did not find a significant relationship between time since surgical treatment of prostate cancer and decision regret, up to 46 months after radical prostatectomy [16].

In the recent Salsburg Statement on Shared Decision Making, international experts called on clinicians to recognize an ethical imperative to share important decision-making with patients, stimulate a two-way flow of information between clinicians and patients, provide accurate information about options and uncertainties, and tailor information to individual patients' needs [17]. Although patient preferences for participation in healthcare decision-making are variable [18], data from our study as well as others [13] suggest that involving patients in decision-making in clinical practice may be associated with reduced decision regret among patients.

Our study is subject to a number of limitations, including the following: a small sample size, a crosssectional design for analysis of decision regret with a lack of prospective follow-up data, limited time duration from decision-making to the measurement of decision regret, the reporting of exploratory secondary analyses, and the reliance on patients' perceptions of RAI treatment decision-making process with no objective information on patient-physician interactions (such as video or audio recordings of the meetings). Our study may have also been subject to some recruitment bias, as participants were recruited as part of a larger study to evaluate a computerized decision aid and therefore could have particularly valued participation in medical decision-making. Also, most individuals in this study were English speaking and had higher education, so the results may not be generalizable to non-English speaking or less educated populations. Finally, we did not collect any data on disease recurrence status or possible thyroid cancer treatment side effects.

In conclusion, the findings from this exploratory, hypothesis-generating study suggest that a lack of patient involvement in healthcare decision-making may be associated with increased decision regret. This hypothesis needs to be validated in a larger prospective study, formally examining decision regret in patients at multiple points over time, ideally with direct examination of patient-physician interactions at the time of decision-making. Further research is also needed to better understand the different ways in which patients may be involved in medical decision-making and its potential impact on future emotional health and wellbeing. Such research may benefit patients and clinicians in all medical and surgical fields, who may be involved in healthcare decision-making.

Acknowledgements This study was supported by an operating grant from the Canadian Institutes of Health Research (Priority Announcement Knowledge Translation (FRN 94609)). This study was also supported, in part, by a University Health Network Thyroid Research Centre Endowment fund. Anna Sawka has been supported, in part, by a New Investigator grant from the Canadian Institutes of Health Research (CNI-80701). Anna Sawka currently holds a Chair in Health Services Research from Cancer Care Ontario, funded by the Ontario Ministry of Health and Long-term Care. Sharon Straus holds a Tier 1 Canada Research Chair. We would like to thank Mrs. Coreen Marino for administrative assistance. We sincerely thank all participants in the study for their time and valued input.

Open Access This article is distributed under the terms of the Creative Commons Attribution Noncommercial License which permits any noncommercial use, distribution, and reproduction in any medium, provided the original author(s) and source are credited. 


\section{References}

1. Liu S, Semenciw R, Ugnat AM, Mao Y (2001) Increasing thyroid cancer incidence in Canada, 1970-1996: time trends and ageperiod-cohort effects. Br J Cancer 85:1335-1339

2. Edwards BK, Howe HL, Ries LA, Thun MJ, Rosenberg HM, Yancik R, Wingo PA, Jemal A, Feigal EG (2002) Annual report to the nation on the status of cancer, 1973-1999, featuring implications of age and aging on U.S. cancer burden. Cancer 94:2766-2792

3. Davies L, Welch HG (2006) Increasing incidence of thyroid cancer in the United States. JAMA 295:2164-2167

4. Colonna M, Grosclaude P, Remontet L, Schvartz C, Mace-Lesech J, Velten M, Guizard A, Tretarre B, Buemi AV, Arveux P, Esteve J (2002) Incidence of thyroid cancer in adults recorded by French cancer registries (1978-1997). Eur J Cancer 38:1762-1768

5. Dos Santos SI, Swerdlow AJ (1993) Thyroid cancer epidemiology in England and Wales: time trends and geographical distribution. Br J Cancer 67:330-340

6. American Thyroid Association (ATA) Guidelines Taskforce on Thyroid Nodules and Differentiated Thyroid Cancer, Cooper DS, Doherty GM, Haugen BR, Kloos RT, Lee SL, Mandel SJ, Mazzaferri EL, McIver B, Pacini F, Schlumberger M, Sherman SI, Steward DL, Tuttle RM (2009) Revised American Thyroid Association management guidelines for patients with thyroid nodules and differentiated thyroid cancer. Thyroid 19:1167-1214

7. Pacini F, Schlumberger M, Dralle H, Elisei R, Smit JWA, Wiersinga W, the European Thyroid Cancer Task Force (2006) European consensus for the management of patients with differentiated thyroid carcinoma of the follicular epithelium. Eur J of Endo 154:787-803

8. British Thyroid Association, Royal College of Physicians (2007) Guidelines for the management of thyroid cancer, 2nd edn. http:// www.british-thyroid-association.org/news/Docs/Thyroid_cancer_ guidelines_2007.pdf Accessed 18 April 2011
9. Sawka AM, Brierley JD, Tsang RW, Thabane L, Rotstein L, Gafni A, Straus S, Goldstein DP (2008) An updated systematic review and commentary examining the effectiveness of radioactive iodine remnant ablation in well-differentiated thyroid cancer. Endocrinol Metab Clin North Am 37:457-480

10. Zeelenberg M, Pieters R (2007) A theory of regret regulation 1.0. J Consum Psychol 17:3-18

11. Sawka AM, Straus S, Gafni A, Brierley JD, Tsang RW, Rotstein L, Ezzat S, Thabane L, Rodin G, Meiyappan S, David D, Goldstein DP (2011) How can we meet the information needs of patients with early stage papillary thyroid cancer considering radioactive iodine remnant ablation? Clin Endocrinol (Oxf) 74:419-423

12. Sawka AM, Meiyappan S, David D, Straus S, Gafni A, Brierley JD, Tsang RW, Rodin G, Rotstein L, Ezzat S, Goldstein DP (2011) A mixed methods evaluation of a computerized decision aid for patients considering radioactive iodine remnant ablation: developing person-centered medicine for thyroid cancer. In press Int J Pers Cent Med

13. Brehaut JC, O'Connor AM, Wood TJ, Hack TF, Siminoff L, Gordon E, Feldman-Stewart D (2003) Validation of a decision regret scale. Med Decis Making 23:281-292

14. Roese NJ, Summerville A (2005) What we regret most... and why. Pers Soc Psychol Bull 31:1273-1285

15. Baike DR, Markman KD, Karadogan F (2009) What we regret most are lost opportunities: a theory of regret intensity. Pers Soc Psychol Bull 35:385-397

16. Lin YH (2011) Treatment decision regret and related factors following radical prostatectomy. Cancer Nurs 34:417-422

17. Seminar SG (2011) Salsburg statement on shared decision making. BMJ 342:d1745

18. Flynn KE, Smith MA, Vanness D (2006) A typology of preferences for participation in healthcare decision making. Soc Sci Med 63:1158-1169 